\title{
NOUVEAUX NÉMATODES ATRACTIDES PARASITES DU CHIMPANZÉ ET DU GORILLE AU GABON
}

\author{
D. VAN WAEREBEkE*, A. G. CHABAUd, J. Y. COLlet
}

\begin{abstract}
RÉSUMÉ. Description de femelles de Probstmayria recueillies dans les matières fécales de Chimpanzés et de Gorilles sauvages, vivant dans la réserve de la Lopé-Okanda au Gabon.

$P$. gombensis du Chimpanzé et $P$. gabonensis $n$. sp. du Gorille ont des structures céphaliques comparables aux autres espèces du genre et ne sont caractérisées que par des caractères mineurs dans les dimensions. Au contraire, $P$. invers $a$ n. sp. du Chimpanzé et $P$. goodallae n. sp. du Gorille ont une structure céphalique aberrante avec une symétrie inversée.

Les particularités remarquables du genre Probstmayria sont analysées.
\end{abstract}

Mots-clés : Atractides. Probstmayria n. spp. Gorilla gorilla. Pan troglodytes. Espèces congénériques.

Évolution des symétries.

\section{New atractid Nematodes parasite of Chimpanzee and Gorilla in Gaboon.}

SUMmaRY. Females of the genus Probstmayria, collected in feces of wild Chimpanzees and Gorillas, living in the Lope-Okanda reserve in Gaboon, are described.

The cephalic structures of $P$. gombensis File, 1976 from Chimpanzee and P. gabonensis n. sp. from Gorilla are similar to those of the other species of the genus. In contrast, the cephalic structures of $P$. inversa n. sp. from Chimpanzee and P. goodallae n. sp. from Gorilla are unusual; they have an inverse symetry (one ventral lip and two latero-dorsal lips). The original characteristics of the genus Probstmayria are analysed.

Key-words: Atractidae. Probstmayria n. spp. Gorilla gorilla. Pan troglodytes. Cogeneric species. Symetries evolution.

Les Nématodes Atractides ont une biologie très aberrante. Les femelles pondent, dans l'intestin de l'hôte, des larves du troisième stade, qui se développent sur place, sans passage par le milieu extérieur. Cette biologie qui aboutit à une multiplication intense du nombre des spécimens, s'effectue dans un organe de grande taille tel que le cæcum des Herbivores.

Muséum National d'Histoire Naturelle, Laboratoire de Zoologie (V'ers), associé au C. N. R. S., 61 , rue Buffon, $F$ 75231 Paris Cedex 05.

* Entomonématologiste O.R.S.T. O.M.

Accepté le 23 novembre 1987. 
Le genre Probstmayria Ransom, 1907, dont la morphologie est particulièrement primitive, compte actuellement sept espèces (voir Van Waerebeke et coll., 1988) réparties chez des hôtes variés; le phénomène, fréquent en parasitologie, de la pluralité d'espèces congénériques chez un même hôte, n'avait pas encore été constaté chez les Atractidae.

L'objet de ce travail est de décrire la coexistence de deux espèces chez le Chimpanzé et de deux espèces chez le Gorille. Dans chacun des deux cas, on trouve une espèce à structure céphalique normale (une lèvre dorsale et deux lèvres latéroventrales) et une deuxième espèce, très proche, mais à structure céphalique inversée (une lèvre ventrale et deux lèvres latéro-dorsales).

Le matériel étudié a été récolté en 1984, dans les matières fécales de Chimpanzés et de Gorilles sauvages vivant dans la réserve de la Lopé-Okanda au Gabon (Station d'Études des Gorilles et des Chimpanzés, Booné, Gabon).

\section{I - Probstmayria gombensis File, 1976}

Hôte : Pan troglodytes L.

\section{Description}

Tête constituée par six lobes saillants en avant, bien individualisés et épais si bien que le diamètre de la tête en vue latérale, dépasse un peu celui de la région cervicale (fig. 1, C). Bouche hexagonale entourée de six papilles labiales internes et, postérieurement, sur les lobes céphaliques de quatre papilles labiales externes, quatre papilles céphaliques et deux amphides (fig. 1, E).

Cavité buccale à section arrondie (fig. 1, F), puis triangulaire (fig. 1, G). La partie antérieure de la cavité buccale est soutenue par une armature chitinoïde complexe (fig. 1, K) : une base globulaire (A) est située dans chacun des 3 axes (dorsal et latéro-ventraux). Elle est prolongée latéralement par 2 petites ailes (B) (fig. 1, G). Ces formations donnent naissance en avant à de petits crochets chitinoïdes : deux très fins (a) insérés sur le globule $A$, deux, un peu plus forts (b) insérés chacun sur une aile $\mathrm{B}$ ( fig. $1, D, F, K$ ). Il en résulte que chaque angle de la bouche est soutenu en profondeur soit par 2 petits crochets (a) issus d'un globule A (angles dorsal et latéro-ventraux), soit par 2 crochets b, issus de 2 lames adjacentes (B) (angles ventral et latéro-dorsaux) (fig. 1, F).

En arrière de cette armature (fig. $1, H$ ), la cavité buccale porte 3 grosses protubérances dans les axes dorsal et latéro-ventraux et 3 fines protubérances dans les axes ventral et latéro-dorsaux. Au fond ( fig. 1, I), les protubérances s'effacent et se creusent en 3 cavités (axes dorsal et latéro-ventraux) et 3 gouttières (axes ventral et latéro-dorsaux).

Pharynx très allongé, cylindrique (fig. 1, B). CEsophage composé d'un corpus, d'un isthme et d'un bulbe bien individualisés. Corpus cylindrique; isthme relativement court, dilaté vers l'avant; bulbe valvulé. Anneau nerveux à l'union du tiers 


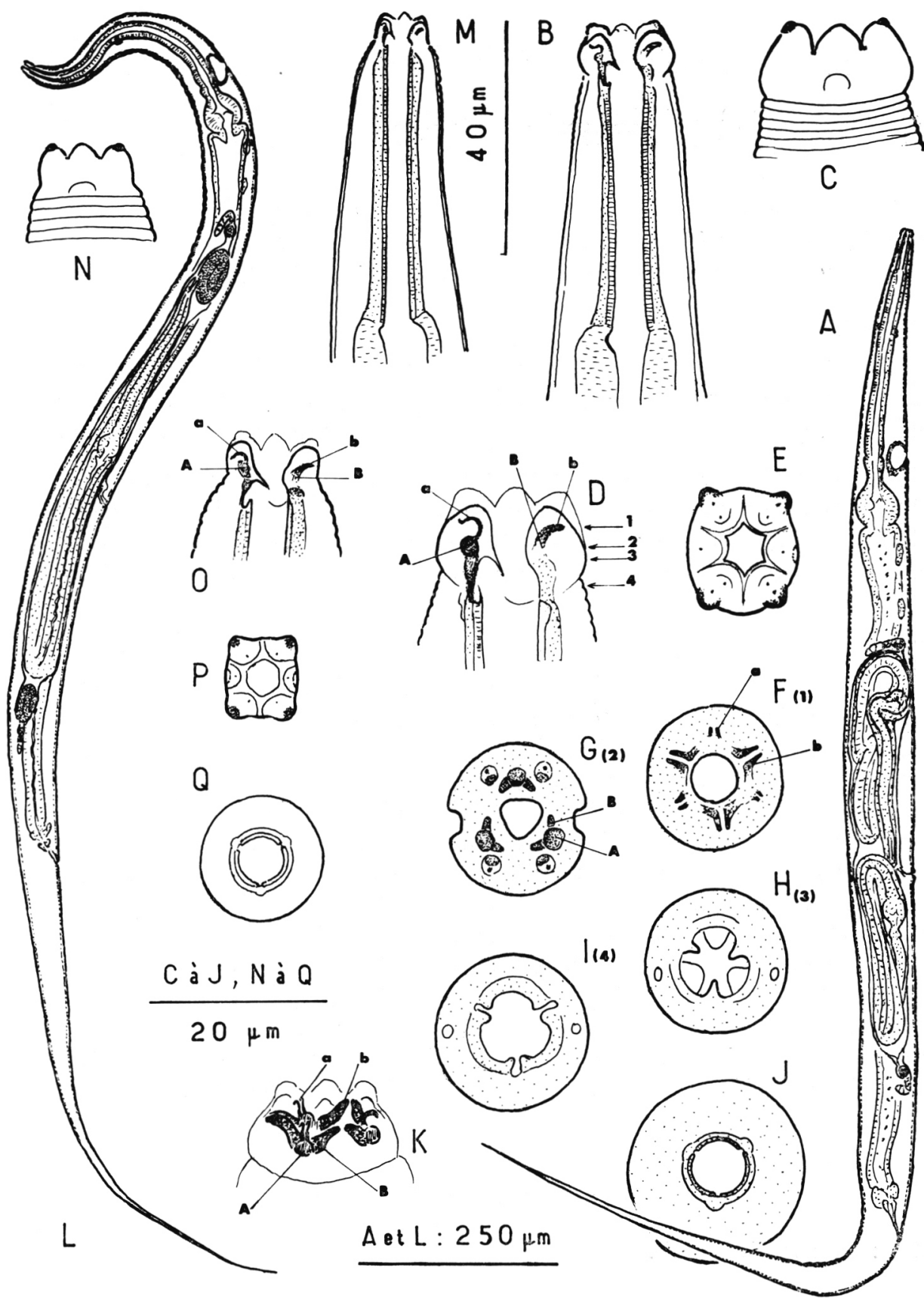

FIG. 1. - A-K : Probstmayria gombensis, femelle.

A : corps, vue latérale droite ; B : extrémité antérieure, vue latérale droite ; $\mathrm{C}$ : tête, vue latérale droite superficielle; $\mathrm{D}$ : tête, vue latérale droite, coupe optique subsagittale, l'axe ventral est à droite, l'axe dorsal à gauche ; $1,2,3,4$ : niveau des coupes optiques figurées ; E : tête, vue apicale superficielle $; \mathrm{F}$ : tête, vue apicale, coupe optique niveau $1 ; G$ : tête, coupe optique niveau 2 ; $\mathrm{H}$ : tête, coupe optique niveau 3 ; I : tête, coupe optique niveau 4 ; $\mathrm{J}$ : coupe transversale au niveau du pharynx ; K : tête, vue apico-latérale gauche montrant l'armature chitinoïde des axes dorsal et latéro-ventraux.

L-Q : Probstmayria gabonensis, femelle.

L : corps, vue latérale droite ; M : extrémité antérieure, vue latérale droite ; $\mathrm{N}$ : tête, vue latérale droite superficielle; $\mathrm{O}$ : tête, vue latérale droite, coupe optique subsagittale ; l'axe ventral est à droite, l'axe dorsal à gauche; $\mathrm{P}$ : tête, vue apicale superficielle; $\mathrm{Q}$ : coupe transversale au niveau du pharynx. 
antérieur et des $2 / 3$ postérieurs du corpus. Pore excréteur à l'union isthme-œsophage débouchant dans une grosse vésicule à parois chitinoïdes épaisses. Vulve pré-équatoriale.

Utérus n'occupant que la partie moyenne du corps, contenant une ou deux larves bien développées et quelques morulas. Amphidelphie. Ovaires très courts avec très peu d'oocytes (de 2 à 4). Queue longue et fine (fig. 1, A).

Dimensions

Holotype : longueur : 1,79 mm; largeur : $81 \mu \mathrm{m}$; anneau nerveux et pore excréteur respectivement à $129 \mu \mathrm{m}$ et $273 \mu \mathrm{m}$ de l'apex ; pharynx : $42 \mu \mathrm{m}$; œsophage : $309 \mu \mathrm{m}$; vulve à $780 \mu \mathrm{m}$ de l'extrémité antérieure ; queue : $570 \mu \mathrm{m}$.

Paratypes (5 spécimens) : longueur : 1,84 $\mu \mathrm{m}(1,79-1,96)$; largeur : $77 \mu \mathrm{m}$ (64-85) ; anneau nerveux et pore excréteur respectivement à $139 \mu \mathrm{m}$ (129-146) et $275 \mu \mathrm{m}$ (265-304) de l'apex ; pharynx : $41 \mu \mathrm{m}$ (40-43) ; œsophage : $304 \mu \mathrm{m}$ (286-317) ; vulve à $789 \mu \mathrm{m}$ (741-905) de l'extrémité antérieure ; queue : $606 \mu \mathrm{m}$ (570-640).

\section{Discussion}

Probstmayria gombensis File, 1976 a été décrit chez des Chimpanzés Pan troglodytes schweinfurthii vivant dans le Gombe National Park en Tanzanie.

Aucune différence morphologique n'apparaît; la structure céphalique en particulier, qui est décrite en détail, correspond bien à celle de notre matériel. Dans les mensurations au contraire, certaines différences sont notables : les longueurs du corps sont presque identiques, mais les spécimens du Gabon ont un pore excréteur plus antérieur : $275 \mu \mathrm{m}$ (265-304) au lieu de $311 \mu \mathrm{m}(300-322)$, une vulve plus antérieure $792 \mu \mathrm{m}$ de l'extrémité antérieure (741-905) au lieu de $851 \mu \mathrm{m}$ (814882) et une queue plus longue : $606 \mu \mathrm{m}$ (570-640) au lieu de $536 \mu \mathrm{m}$ (520-580).

Les meilleurs caractères différentiels entre les espèces de Probstmayria sont fournis par les spicules et le gubernaculum; n'ayant pas les mâles du Gabon, il est difficile de savoir si nous avons une ou deux espèces. Nous préférons donc provisoirement utiliser le nom de gombensis, en supposant que les différences constatées sont liées à l'existence de deux populations différentes.

\section{II - Probstmayria inversa n. sp.}

Hôte : Pan troglodytes L.

\section{Description}

Tête grosse et plate, séparée du corps par un cou perceptible en vue latérale (fig. 2, C).

Bouche triangulaire, limitée par trois pseudo-lèvres, une ventrale et deux latéro-dorsales, renforcées chacune par un épaississement chitinoïde (fig. 2, D). 
Six papilles labiales internes. Amphides et papilles céphaliques situées sur un cycle plus postérieur. Papilles labiales externes non perceptibles.

La cavité buccale ( $f i g .2, E, F$ ) est occupée en avant par trois lobes charnus très légèrement dentés à l'apex sur les axes ventral et latéro-dorsaux. Les trois axes dorsal et latéro-ventraux au contraire, sont creusés en gouttière (fig. 2, G). Plus en arrière, la cavité buccale est triangulaire avec une légère armature chitinoïde aux trois angles dorsal et latéro-ventraux (fig. $2, H$ ). C'est seulement au fond de la cavité buccale que la symétrie normale apparaît (une paroi dorsale et deux parois latéro-ventrales). Dans cette zone, chaque paroi porte un tubercule central et deux petites lames latérales (fig. 2, I), puis les tubercules disparaissent et il ne subsiste que les six petites lames, libres dans la lumière buccale (fig. $2, J$ ).

Pharynx très court. Anatomies digestive et génitale conformes à celles des autres espèces du genre.

Dimensions

Holotype : longueur : 2,3 mm; largeur : $119 \mu \mathrm{m}$; anneau nerveux et pore excréteur respectivement à $163 \mu \mathrm{m}$ et $370 \mu \mathrm{m}$ de l'apex ; pharynx : $19 \mu \mathrm{m}$; œsophage : $443 \mu \mathrm{m}$; vulve à $990 \mu \mathrm{m}$ de l'extrémité antérieure ; queue : $721 \mu \mathrm{m}$.

Les autres spécimens disponibles sont rétractés et n'ont pas pu être mesurés.

\section{Discussion}

Ce Nématode se sépare aisément des autres espèces de Probstmayria déjà connues, d'une part par la structure particulière de la tête et de la capsule buccale et d'autre part par la petite taille du pharynx $(19 \mu \mathrm{m})$. Cet organe a une longueur allant de 35 à $170 \mu \mathrm{m}$ chez les autres espèces de Probstmayria. Nous avons donc une espèce nouvelle que nous nommons Probstmayria inversa n. sp., en raison de la bouche ayant une symétrie inverse de celle des autres espèces.

\section{III - Probstmayria gabonensis n. sp.}

Hôte : Gorilla gorilla L.

\section{DESCRIPTION}

Les structures générales et, en particulier, celles de la tête et de la capsule buccale sont identiques à celles de $P$. gombensis. L'extrémité céphalique ( fig. $1, O, P, Q$ ) est cependant nettement plus fine ; en vue latérale, les lèvres sont plates et non convexes comme dans les autres espèces (fig. $1, \mathrm{~N}$ ).

\section{Dimensions}

Holotype : longueur : 1,96 mm; largeur : $68 \mu \mathrm{m}$; anneau nerveux et pore excréteur respectivement à 137 et $278 \mu \mathrm{m}$ de l'apex ; pharynx : $42 \mu \mathrm{m}$; œesophage : $298 \mu \mathrm{m}$; vulve à $843 \mu \mathrm{m}$ de l'extrémité antérieure ; queue : $639 \mu \mathrm{m}$. 

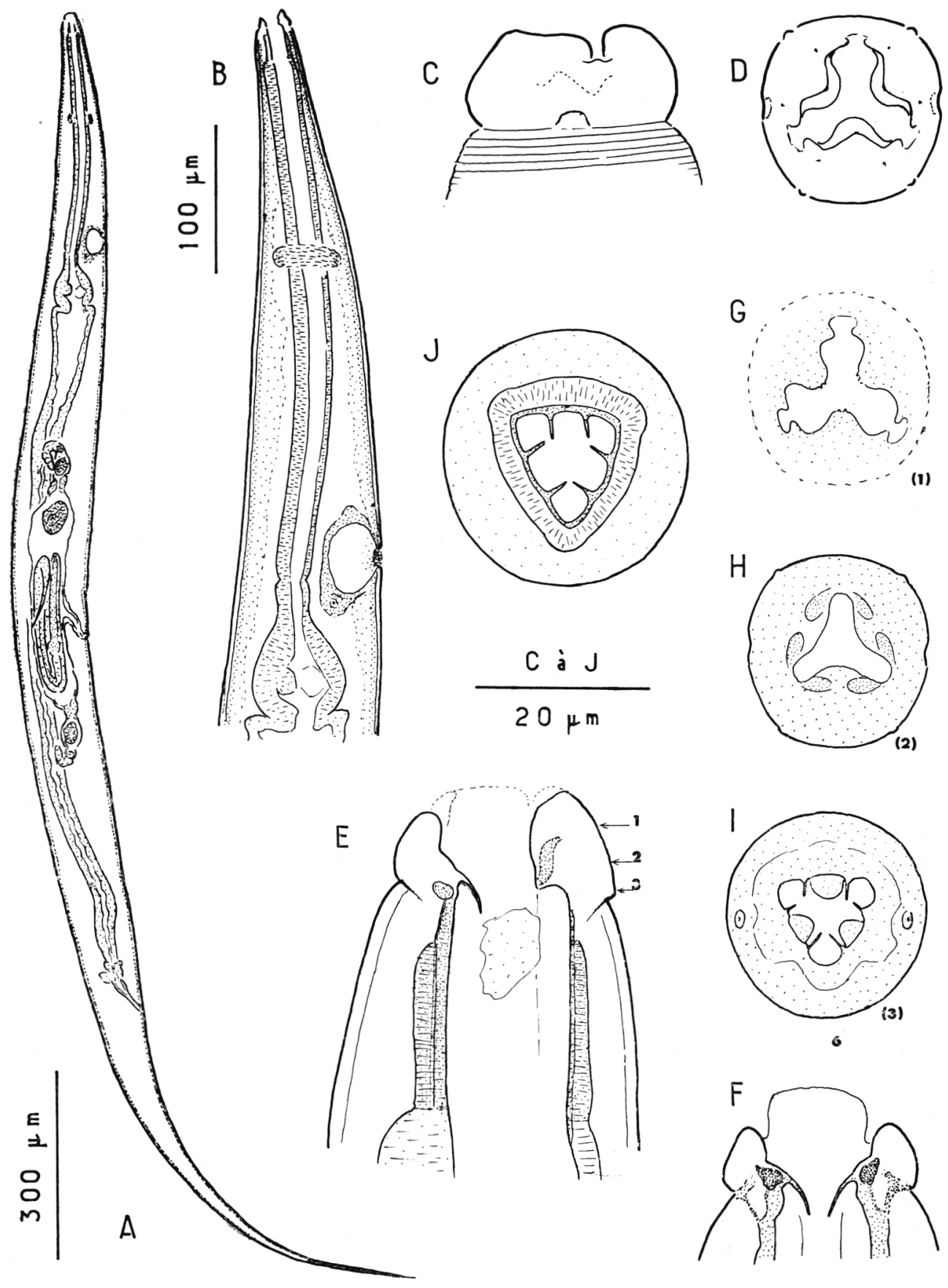

Fig. 2. - Probstmayria inversa, femelle.

A : corps, vue latérale droite ; $\mathrm{B}$ : région œsophagienne, vue latérale droite ; $\mathrm{C}:$ tête, vue latérale droite superficielle; D : tête, vue apicale superficielle ; $\mathrm{E}$ : tête, vue latérale droite, coupe optique subsagittale, l'axe ventral est à droite, l'axe dorsal à gauche; 1,2 , 3, niveau des coupes optiques figurées ; F : tête, lèvre ventrale et coupe optique des lobes latéro-ventraux ; $\mathrm{G}$ : tête, coupe optique niveau $1 ; \mathrm{H}$ : id., coupe optique niveau $2 ; \mathrm{I}$ : id., coupe optique niveau $3 ; \mathbf{J}$ : id., coupe transversale au niveau du pharynx. 
Paratypes (5 spécimens) : longueur : 1,94 mm (1,85-2,18); largeur : $77 \mu \mathrm{m}$ (68-91) ; anneau nerveux et pore excréteur respectivement à $139 \mu \mathrm{m}$ (130-150) et $279 \mu \mathrm{m}$ (263-306) de l'apex ; pharynx : $42 \mu \mathrm{m}$ (39-46) ; œsophage : $297 \mu \mathrm{m}$ (280-321) ; vulve à $830 \mu \mathrm{m}$ (764-907) de l'extrémité antérieure ; queue : $676 \mu \mathrm{m}$ (616-787).

\section{Discussion}

Il serait tentant d'assimiler l'espèce à $P$. gorillae Kreis, 1955, décrit chez Gorilla gorilla et Hylobates concolor au parc zoologique de Bâle.

Cependant, la longueur du corps étant comparable, on constate que la largeur de la tête est plus faible $(10 \mu \mathrm{m}$ au lieu de 15-17 $\mu \mathrm{m})$, l'œesophage est plus court (la jonction œsophage-intestin est à $349 \mu \mathrm{m}$ de la tête au lieu de $394 \mu \mathrm{m}$ ), la queue est plus longue.

Il y a donc de fortes différences dans les mensurations et, par ailleurs, l'espèce de Kreis provenant de deux hôtes différents d'un parc zoologique, il est impossible de savoir si l'infestation décrite provient originellement d'un Gorille ou d'un Gibbon.

L'assimilation de nos spécimens à $P$. gorillae risque donc d'entraîner de graves confusions.

L'espèce se distingue de $P$. gombensis, parasite de Chimpanzé, par la tête plus fine et les lèvres non convexes en vue latérale.

Les autres espèces parasites de Primates sont :

- P. simiae Maplestone, 1931, parasite d'Hylobates au parc zoologique de Calcutta, qui a une vulve équatoriale à lèvres proéminentes, des lèvres céphaliques convexes et une queue assez courte $(450-500 \mu \mathrm{m})$.

- P. nainitalensis Arya, 1981, parasite de Macaca mulatta de Nainital (Inde) qui a une vulve postéquatoriale, des lèvres céphaliques convexes, un pharynx très long $(160-170 \mu \mathrm{m})$, et une queue courte $(470-500 \mu \mathrm{m})$.

L'espèce décrite ci-dessus est donc considérée comme nouvelle et nous la nommons Probstmayria gabonensis n. sp.

\section{IV - Probstmayria goodallae n. sp.}

Hôte : Gorilla gorilla L.

\section{DESCRIPTION}

La bouche, triangulaire, avec un bord ventral et deux bords latéro-dorsaux, est située sur un plateau hexagonal avec les six angles (deux latéraux et quatre latéro-médians) prolongés en pointe (fig. 3,F). Les six papilles du cycle labial interne sont éloignées de la bouche ; les quatre papilles céphaliques et les deux amphides sont situées à l'apex des six pointes saillantes. La cavité buccale conserve 

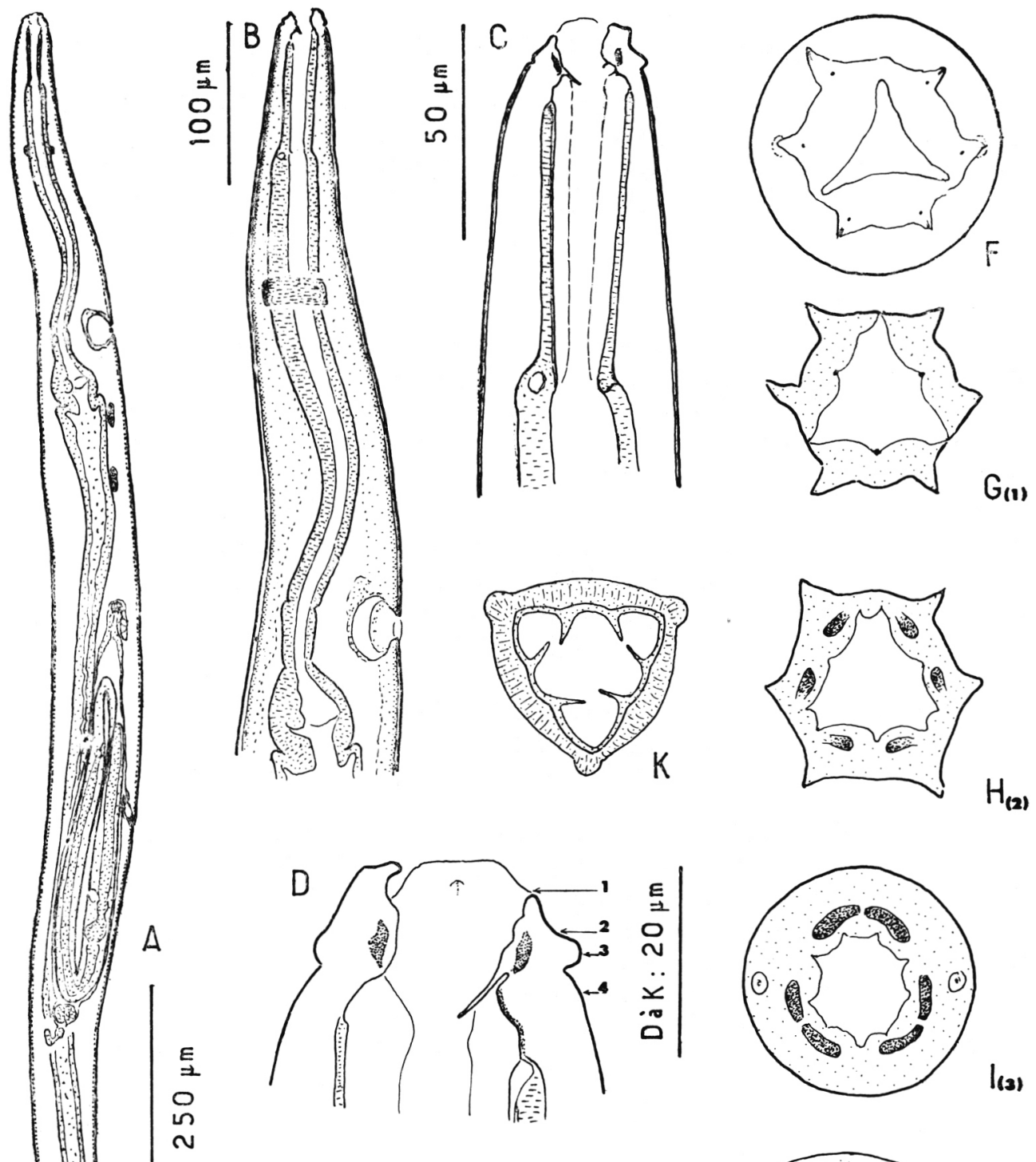

Fig. 3. - Probstmayria goodallae, femelle.

A : corps, vue latérale droite; $\mathrm{B}$ : région œsophagienne, vue latérale droite ; $\mathrm{C}$ : extrémité antérieure, vue latérale droite ; D : tête, vue latérale gauche, coupe optique subsagittale ; 1, 2, 3, 4, niveau des coupes optiques figurées; $\mathrm{E}$ : tête, vue latérale gauche superficielle ; $\mathrm{F}$ : tête, vue apicale superficielle ; $\mathrm{G}$ : tête, coupe optique niveau $1 ; \mathrm{H}$ : id., coupe optique niveau 2 ; $\mathrm{I}$ : id., coupe optique niveau $3 ; \mathbf{J}$ : id., coupe optique niveau $4 ; \mathrm{K}$ : coupe transversale au niveau du pharynx. 
en avant la symétrie inversée de la bouche (fig. 3, G, H). Le fond de la cavité buccale, armé d'une armature chitinoïde, prend une symétrie normale avec une paroi dorsale et deux parois latéro-ventrales. Chaque paroi porte en avant un tubercule central, bordé d'une paire de lamelles saillantes dans la lumière (fig. 3, I). Au fond de la cavité, les tubercules ont disparu et seules les pointes des lamelles restent visibles (fig. $3, J$ ). Pharynx allongé. Anatomies digestive et génitale conformes à celles des autres espèces du genre.

Dimensions

Holotype : longueur : 2,01 mm; largeur : $101 \mu \mathrm{m}$; anneau nerveux et pore excréteur respectivement à 171 et $382 \mu \mathrm{m}$ de l'apex ; pharynx : $64 \mu \mathrm{m}$; œsophage : $366 \mu \mathrm{m}$; vulve à $907 \mu \mathrm{m}$ de l'extrémité antérieure ; queue : $602 \mu \mathrm{m}$.

Paralypes (2 spécimens) : longueur : ?-1,93 mm ; largeur : 99-84 $\mu \mathrm{m}$; anneau nerveux et pore excréteur respectivement à 175-165 $\mu \mathrm{m}$ et 348-352 $\mu \mathrm{m}$ de l'apex ; pharynx : $68-64 \mu \mathrm{m}$; assophage : $377-345 \mu \mathrm{m}$; vulve à $894-815 \mu \mathrm{m}$ de l'extrémité antérieure; queue : ?-611 $\mu \mathrm{m}$.

\section{Discussion}

L'inversion de la symétrie céphalique s'accompagne d'une certaine atrophie des lobes labiaux, si bien que l'extrémité antérieure, en vue latérale, est relativement large et plate. Ce caractère est donc décelable même en l'absence d'études en vue apicale. Il en résulte que la seule espèce ayant un aspect comparable de l'extrémité antérieure est $P$. inversa décrit plus haut.

$P$. inversa diffère par le pharynx très court $(19 \mu \mathrm{m}$ au lieu de $40 \mu \mathrm{m})$ et par la structure buccale dépourvue des six pointes saillantes caractérisant notre quatrième espèce.

Celle-ci est donc nouvelle et nous la nommons Probstmayria goodallae n. sp., en respectueux hommage au Docteur Jane Goodall.

\section{Discussion générale}

Le genre Probstmayria Ransom, 1907 et le genre Fitzsimmonsnema Petter, 1966, qui n'en diffère que très peu, constituent un groupe très remarquable pour plusieurs raisons :

- Parasitisme chez des hôtes très différents.

Les hôtes actuellement connus comprennent : Tortues terrestres, Tapirs du nouveau monde, Équidés, Suidés et Primates de l'ancien monde.

- Homogénéité morphologique et biologique.

En dehors des structures céphaliques aberrantes des deux espèces décrites ci-dessus, toutes les espèces sont morphologiquement presque identiques. Elles 
ne diffèrent entre elles que par des détails dans les mensurations ou dans les caractères des spicules et du gubernaculum.

Du point de vue biologique, toutes les espèces présentent une poeciandrie : les mâles sont rares ou très rares.

\section{- Ancienneté du groupe.}

Toutes les espèces proviennent d'Afrique ou d'Asie sauf une espèce néotropicale chez le Tapir. Celle-ci n'a aucune particularité morphologique très marquée. Étant donné l'isolement de l'Amérique du Sud durant l'ère tertiaire, une forme très proche des formes actuelles a donc dû exister à une période géologique très reculée (Van Waerebeke et coll., 1987).

\section{- Évolution de la morphologie céphalique.}

Le changement de symétrie céphalique constaté chez deux des espèces étudiées plus haut n'est pas un phénomène unique chez les Nématodes.

Chez certains Ascaridida, les lobes interlabiaux insérés entre les trois lèvres primitives peuvent prendre un grand développement (pseudo-lèvres). Chez les Oxyurida, certains genres atteignent un stade de véritable inversion de la symétrie céphalique. Dans la lignée Heteromyoxyuris - Evaginuris - Helminthoxys analysée par Quentin (1973), on assiste à une transformation graduelle des structures conduisant, comme chez Probstmayria, à des structures superficielles totalement inversées. Un fait notable chez Probstmayria est donc que l'on ne connaît pas d'intermédiaire entre la structure très homogène de presque toutes les espèces et les structures très aberrantes de $P$. inversa et de $P$. goodallae.

\section{- Processus de spéciation envisageable.}

Les analyses phénétiques ou cladistiques conduiraient à isoler $P$. inversa et $P$. goodallae dans un groupe particulier. Cela s'oppose à la plupart des exemples connus de spéciations multiples chez les Nématodes parasites de Vertébrés. On constate en effet que dans les cas de parasitisme par plusieurs espèces congénériques, les espèces qui se trouvent chez un même hôte sont plus proches entre elles qu'elles ne le sont des espèces d'un hôte voisin. En d'autres termes, la faune se constitue par spéciations successives chez un même hôte et non par emprunts à des hôtes voisins (Chabaud et Durette-Desset, 1978). Par analogie avec ce qui se passe dans les cas plus facilement analysables, nous pensons donc plus volontiers que $P$. inversa dérive de $P$. gombensis et que $P$. goodallae dérive de $P$. gabonensis.

\section{BIBLIOGRAPHIE}

Arya S. N. : A new species of the Genus Probstmayria Ransom, 1907 (Nematoda: Atractidae) from the Rhesus macaque, Macaca mulatta. Primates, 1981, 22, 261-265.

Chabaud A. G., Durette-Desset M.-C. : Parasitisme par plusieurs espèces congénériques. Bull. Soc. Zool. Fr., 1978, 103, 459-464.

File S. K. : Probstmayria gombensis sp. n. (Nematoda: Atractidae) from the Chimpanzee. J. Parasitol., 1976, 62, 256-258. 
KreIs H. A. : Beiträge zur Kenntnis parasitischer Nematoden. XVIII. Das genus Probstmayria Ransom, 1907. Schweiz. Arch. Tierheilkd., 1955, 97, 422-433.

Maplestone P. A. : Parasitic Nematodes obtained from animals dying in the Calcutta Zoological Gardens. Pt. 4-8. Rec. Indian Mus., 1931, 33, 71-171.

Petter A. J. : Équilibre des espèces dans les populations de Nématodes parasites du côlon des Tortues terrestres. Mem. Mus. Natn. Hist. Nat., Paris, série A, Zool., 1966, 39, 1-252.

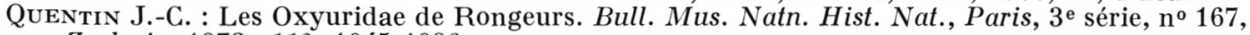
Zoologie, 1973, 112, 1045-1096.

Ransoni B. H. : Probstmayria vivipara (Probstmayr, 1865) Ransom, 1907, a nematode of horses herefore unreported from the United States. Trans. Am. Micr. Soc., 1907, 27, 33-40.

Van Waerebeke D., Chabaud A. G., Anthony G. : Probstmayria tapiri n. sp., Nématode parasite d'un Tapir du Nouveau Monde. Bull. Mus. Natn. Hist. Nat., Paris, 1988 (sous presse) 